\title{
Performance Enhancement of Distributed System through Load Balancing and Task Scheduling
}

\author{
Abhijit A. Rajguru \\ Research Scholar at Walchand Institute of \\ Technology \\ Solapur, Maharashtra, India
}

\author{
Sulabha S. Apte, PhD \\ WIT, Solapur \\ Maharashtra, India
}

\begin{abstract}
Task scheduling \& load balancing in distributed network are the most challenging research area in computer science. In distributed systems, scheduling mechanism has more issues as there is no centralized authority to allocate the workload among multiple processors. Further, handling both load balancing and scheduling at hand is a daunting task. In this paper, we propose a fuzzy based load balancing and task scheduling technique to optimize the performance of distributed system. Initially, clusters are formed and node with larger buffer availability and high CPU speed is elected as cluster head. Tasks are prioritized into flexible and non-flexible using task prioritizing strategy. Non-Flexible tasks are prioritized over flexible tasks. For non-flexible tasks, essential information of nodes such as CPU speed, work load and distance from cluster head are made pass through the fuzzy system. Node state is obtained as output. Based on states of node, non-flexible tasks are allocated. Our technique dynamically handles scheduling and load balancing at hand. We use simulation results to prove efficiency of our technique.
\end{abstract}

\section{Keywords}

Distributed Computing, Fuzzy Logic, Load Balancing, Task Scheduling.

\section{INTRODUCTION}

\subsection{Distributed Systems}

A distributed system consists of set of communication and computing resources, which are shared by active users. [1] In other words, a large loosely coupled distributed system is formed by connecting a group of work stations through the communication channel. [2] Resource sharing is the foremost advantage of distributed system. Reliability, scalability, economy, inherent distribution and functional separation are the features of distributed system. [3]

On account of time accuracy and other features of distributed system, it is mainly useful in telecommunication networks such as telephone networks and computer networks (Internet). Distributed system has many application like aircraft control system, industrial control systems, multiplayer online games and virtual reality etc.[4]

\subsection{Load balancing}

In parallel \& distributed systems, multiple programs are processed parallelly by multiple processors. The quantity of time assigned to a processor to execute task is termed as work load of a processor. [5] The process of equalizing loads among the processors is known as load balancing. This technique attains good throughput without the requirements of additional hardware. [6]

Load balancing can be achieved statically (at the beginning) or dynamically (run time). Based on this, it is classified into two types as static load balancing and dynamic load balancing. [7]

\section{A. Static Load- balancing}

Static load balancing balances the load of system using priori knowledge of applications and statistical knowledge about the system. The entire process is performed by considering average behavior of system.

\section{B. Dynamic load-balancing}

In dynamic load balancing technique, loads are allocated to work stations dynamically to balance the work load. This process is continued until processes are completed or terminated. New jobs may be added to the cluster of machines at any time by the user and are scheduled by load balancing system. [8]

The problem of load balancing becomes significant as demand for computing power increases. The main objective of load balancing is to improve the distributed system performance by means of allocating loads appropriately. [7] In parallel and distributed systems, load balancing is considered to be a critical issue for it has to assure fast processing and good utilization. [9]

\subsection{Scheduling in distributed systems}

Apart from load balancing, task scheduling is also an important technique to improve the performance and throughput of distributed network. [10] The process of distributing tasks among processors is defined as scheduling. Task scheduling is differentiated into local scheduling and global scheduling.[5]

\section{A. Local scheduling}

This technique allocates the processes to the time slices of the processor.

\section{B. Global scheduling}

Global scheduling makes a decision of place to execute a process in the multiprocessor system. Here, the scheduling is accomplished either by a central/ master device or may be distributed among the processing elements. Global scheduling is further divided into two types as static scheduling and dynamic scheduling[5].

In static scheduling processes are assigned to processors before execution starts. On other hand dynamic scheduling can reassign the processes to the processor during execution.

\subsection{Issues of load balancing and scheduling}

The load balancing and scheduling mechanism in distributed systems has various issues as there is no centralized authority to allocate the workload among multiple processors. Some of the issues are described below:

1) Achieving load balancing in the network is difficult, as processes move from a node to another amidst execution of work load. [5]

2) The diverse nature of both huge users and computing resources present more complicated challenges in scheduling and processing resources. [11] 
3) An efficient load balancing scheme should support characteristics such as stable, scalable and low overhead. However, these characteristics are interdependent in nature. [7]

4) Handling both scheduling and load balancing at hand is a daunting task. [10] Further, the main attributes of scheduling algorithm namely fairness and locality often conflict each other. [12]

\subsection{Problem Identification}

Till date, the literature has enormous work for load balancing and task scheduling in distributed system. But they did not concentrate on the task requests which may be flexible during task allocation.

Resource aware distributed scheduling strategies for distributed systems are described in [11]. In this work, the major consideration is provided to sink nodes and not to source nodes. Also, the sink nodes are selected based on buffer information alone. But there are other factors which influence the task scheduling like distance between each cluster members, workload and deadline of each task etc. They do not use any specific mechanism to elect the coordinator node (cluster head).

To overcome the above described problems, we propose to develop a fuzzy based load balancing and task scheduling technique for distributed system which provides more accuracy and efficiency.

\section{RELATED WORK}

Daniel Grosu et al [1] have presented a game theoretic framework for obtaining a fair load balancing scheme. Their main goal was to derive a fair and optimal allocation scheme. They have formulated the load balancing problem in single class job distributed systems as a cooperative game among computers and it is also a fair solution. In [7] the same authors have used a game theoretic frame work for obtaining a fair balancing scheme. In this case they have formulated the load balancing problem in heterogeneous distributed systems as a noncooperative game among users. In both cases they have showed that the Nash Bargaining Solution (NBS) of this game provides a Pareto optimal operation point for the distributed system.

M. Nikravan et al. [10] have presented a new method for process scheduling in distributed systems. The scheduling in distributed systems is known as an NP-complete problem even in the best conditions, and methods based on heuristic search have been proposed to obtain optimal and suboptimal solutions. In this paper, they have used the power of genetic algorithms for balancing the load efficiently.

Sivakumar et al. [11] have proposed distributed algorithms referred to as Resource-Aware Dynamic Incremental Scheduling (RADIS) strategies. These strategies are specifically designed to handle large volumes of computationally intensive arbitrarily divisible loads submitted for processing at cluster/grid systems involving multiple sources and sinks (processing nodes). The design of these strategies adopts the divisible load paradigm, referred to as the divisible load theory (DLT).

Michael Isard et al. [12] have introduced a powerful and flexible new framework for scheduling concurrent distributed jobs with fine-grain resource sharing. In that, the scheduling problem is mapped to a graph data structure, where edge weights and capacities encode the competing demands of data locality, fairness, and starvation-freedom, and a standard solver computes the optimal online schedule according to a global cost model.

Xuan Lin et al. [13] have addressed the problem of providing deterministic QoS to arbitrarily divisible applications executing in a cluster. Four contributions are made. First, they have extent DLT to compute the minimum number of processors required to meet an application deadline. Second, based on this, a novel algorithmic approach integrating DLT and EDF scheduling has been proposed. Third, important design parameters are identified that affect the performance of real-time divisible-load scheduling algorithms. Finally, they systematically investigated the effects of these design parameters on a set of real-time scheduling algorithms.

Kento Aida et al. [14] have investigated the scheduling of mixedparallel applications, which exhibit task and data parallelism, in advance reservations settings. They have defined two scheduling problems, RESSCHED and RESSCHEDDL, depending on whether the goal is to minimize application turn around- time or to meet a deadline, respectively. For each problem, they have proposed and evaluated a set of scheduling algorithms. These algorithms were compared for both their performance (i.e., turnaround-time and tightest deadline met) and their resource consumption.

Ananda Basu et al. [15] have proposed a scheduling mechanism for distributed system. Their proposed method is based on performing model checking for knowledge properties. It allows identifying where the local information of a process is sufficient to schedule the execution of a high priority transition. As a result of the model checking, the program is transformed to react upon the knowledge it has at each point. The transformed version has no priorities, and uses the gathered information and its knowledge to limit the enabled ness of transitions so that it matches or approximates the original specification of priorities.

\section{FUZZY BASED LOAD BALANCING \& TASK SCHEDULING TECHNIQUE}

\subsection{Overview}

In this paper, we propose a fuzzy based load balancing and task scheduling technique in distributed systems. Our proposed network contains a set of client (C) and server (S) nodes. These nodes form clusters and node with larger buffer and high CPU speed is elected as cluster head $(\mathrm{CH})$. The elected $\mathrm{CH}$ is responsible for coordinating and scheduling tasks. Tasks are prioritized using a Boolean value. Flexible tasks are represented by Boolean value 1 and value 0 denotes non-flexible tasks. When $\mathrm{C}$ nodes receive a new task, it forwards the task details to $\mathrm{CH}$. The $\mathrm{CH}$ looks for flexible field, for non-flexible tasks, it collects information such as work load, CPU speed and distance from $\mathrm{CH}$. These values are made pass through the fuzzy system. Fuzzification is performed by representing input and output variables in membership functions. Fuzzy rules are evaluated and as an outcome of fuzzy system, S' nodes are classified into three states as, schedulable (SC), likely to be schedulable (LS) and not schedulable (NS). Non-flexible tasks are allocated to S' nodes in schedulable state and when buffer requirement exceeds the buffer availability of SC state nodes, then they are allocated to S' nodes in LS state. The flexible tasks are allocated to S' nodes in likely to be scheduled (LS) state.

\subsection{Computation of Metrics}

\subsubsection{Available Buffer Estimation}

The buffer availability of node ' $\mathrm{n}$ ' at time' $\mathrm{t}+1$ ' can be calculated as follows,

$$
A v a B_{n i}^{t+1}=\left(\frac{\sum_{n=0}^{I-1}\left((I-n) \cdot A v a B_{n i}^{t-n}\right.}{\sum_{n=0}^{I-1} n}\right) \cdot p
$$


Where, I denote iteration number, $\mathrm{n}$ stands for node in the network, $A v a B_{n i}^{t-n}$ is the available buffer computed previously at $\mathrm{t}-\mathrm{x}$ and $\mathrm{p}$ is buffer estimating probability, usually set to 0.95 .

\subsubsection{Work load calculation}

Work load $\left(\mathrm{W}_{\mathrm{L}}\right)$ at a node is calculated as follows,

$$
\mathrm{W}_{\mathrm{Li}}=\mathrm{N}_{\mathrm{T}} \times \mathrm{S}_{\mathrm{T}}
$$

Here, $\mathrm{N}_{\mathrm{T}}$ is the number of tasks to be computed and $\mathrm{S}_{\mathrm{T}}$ size of tasks.

\subsection{Network Architecture}

Consider the distributed system comprises of set of client nodes (C's) and server nodes (S's). Client nodes (C's) are nodes that have tasks to be processed by server nodes (S's). Both client and server nodes are formed clusters. Each cluster has a cluster head $(\mathrm{CH})$, it is responsible for coordinating and scheduling tasks. The network architecture is given below in figure-1.

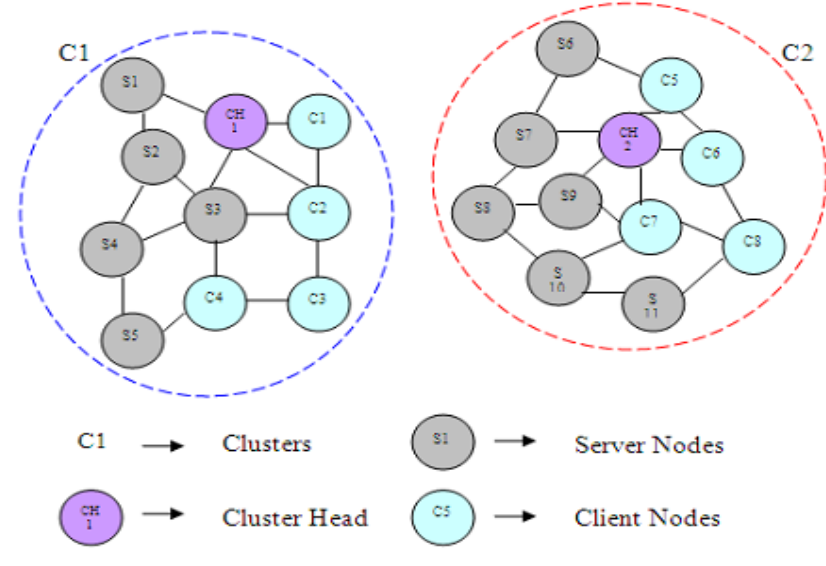

Figure 1: Network Architecture

\subsubsection{Cluster Head Selection}

In each cluster, a cluster head $(\mathrm{CH})$ is elected considering larger buffer size and high CPU speed. The step by step process of cluster head $(\mathrm{CH})$ selection is given below,

(i) After the deployment of nodes in the network, both C's and S's form clusters.

(ii) Within the cluster, each node estimated available buffer value ( $\left.A v a B_{n i}^{t-n}\right)$ using equation (1)

(iii) Each node broadcasts $\mathrm{Nw}$ Info (Network Information) message in the cluster. Nw Info message includes value and CPU speed.

$$
A v a B_{n i}^{t-n}
$$

Node $\stackrel{N w \text { Info }}{\longrightarrow}$ Cluster

(iv) Each node receives the broadcasted value and finally a

$$
\begin{aligned}
& \text { node with larger buffer size ( } \left.A v a B_{n i}^{t-n}\right) \text { and high CPU } \\
& \text { speed is selected as cluster head }(\mathrm{CH}) \text {. }
\end{aligned}
$$

(v) The selected $\mathrm{CH}$ broadcasts this selection information to all nodes in the cluster.

\footnotetext{
$\mathrm{CH} \stackrel{\text { CHselecion }}{\longrightarrow}$ Nodes in the $\mathrm{CH}$
}

The selected $\mathrm{CH}$ is responsible for controlling, coordinating and scheduling the tasks among C's and S's nodes.

\subsection{Task Prioritization Strategy}

The C's nodes contain tasks to be computed and these tasks are distributed among S's nodes by the $\mathrm{CH}$. In real time situations, the task contains stringent deadline requirements, which should be satisfied to avoid unsolicited packet losses. To bring this consideration in our technique we use task prioritization strategy. By this, each task is marked and prioritized using Boolean value.

In general, each task consists of unique task id, task size and deadline (execution time) fields. In addition to these fields, an extra Boolean field is added to specify the priority of tasks. The Boolean value 1 represents flexible and 0 represents non-flexible. We set priority to non-flexible task over flexible. For flexible task, we can extend its execution time (dead line) when its deadline is missed. But, in the case of non- flexible tasks, deadline should not be missed and the tasks have to be processed immediately. The header of task is shown in table-1.

Table-1 Fields in Task Header

\begin{tabular}{|l|l|l|l} 
Task id & Task Size & Deadline & Flexible/ Non-flexible
\end{tabular}

\subsection{Fuzzy Based Task Scheduling Technique}

When C's have new task to be processed, it sends task details to the $\mathrm{CH}$. While receiving task details, the $\mathrm{CH}$ looks for flexible field of task. If the value is zero then $\mathrm{CH}$ gathers work load, $\mathrm{CPU}$ speed and distance from the $\mathrm{CH}$ information from S's nodes of its cluster. Work load of node is estimated as per equation (2). These informations of each server node (S) are made pass through the fuzzy system to predict the states of S nodes. As an outcome of fuzzy system, a server node (S) would be in any of the following three states as schedulable (SC), likely to be schedulable (LS) and not schedulable (NS).

\subsubsection{Fuzzification}

In this phase, the input variables work load, CPU speed and distance from the scheduler are fuzzified by representing in membership functions. The membership functions for input variables work load, CPU speed and distance from the scheduler are given in figure-2, 3 and 4 respectively. Figure-5 represents the membership function of output variable.

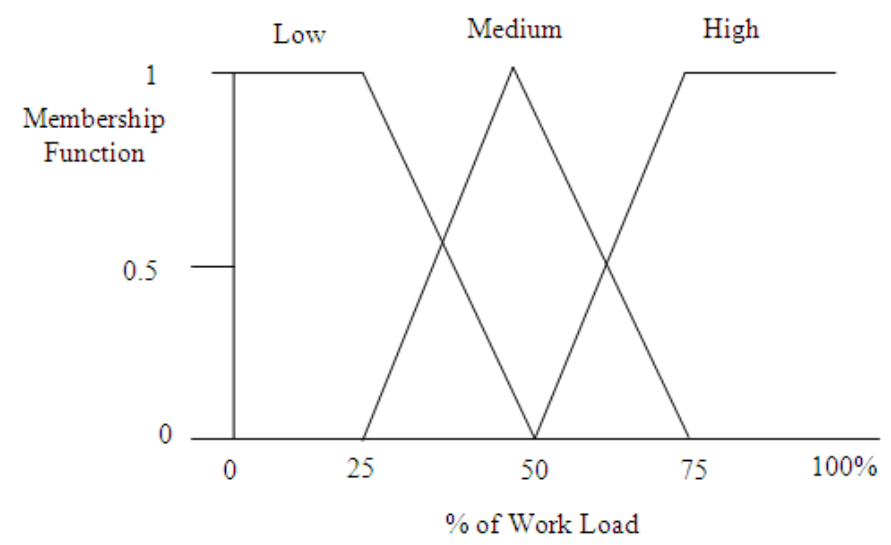

Figure 2: Fuzzy Set for Work Load 


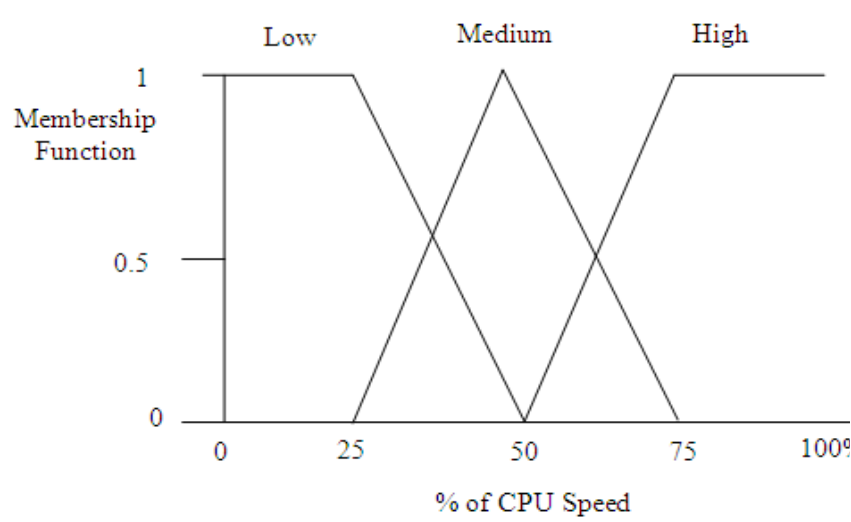

Figure 3: Fuzzy Set for CPU Speed

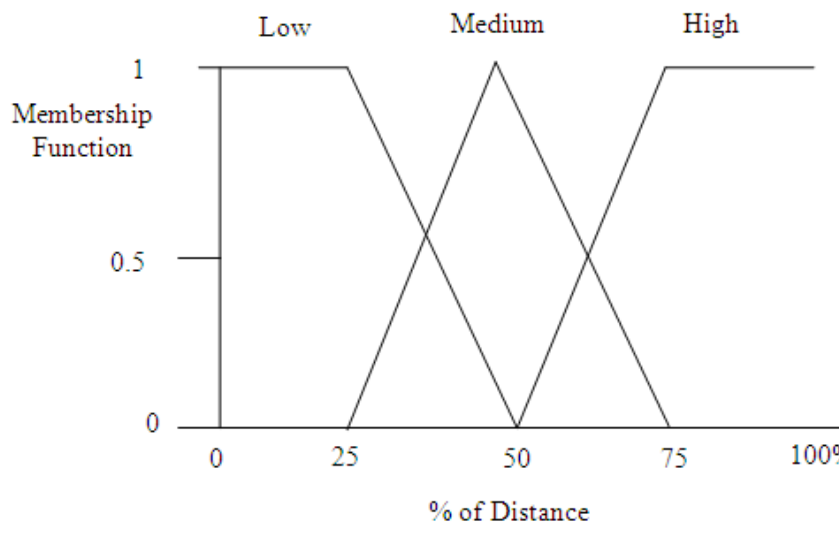

Figure 4: Fuzzy Set for Distance

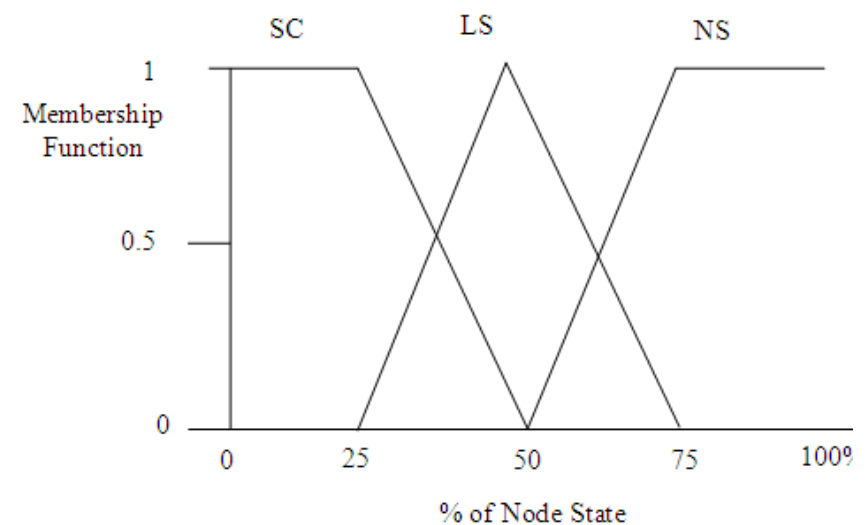

$\mathrm{SC} \rightarrow$ Schedulable $\mathrm{LS} \rightarrow$ Likely Schedulable

$$
\text { NS } \rightarrow \text { Not Schedulable }
$$

Figure 5: Fuzzy Set for Node State

\subsubsection{Fuzzy Inference Engine}

In fuzzy logic system, the fuzzy rules make up the fuzzy inference system. The defined fuzzy rules associates input, output parameters with membership functions. Fuzzy rules take if then else rule format. In our system, fuzzy inference system is constructed considering the following 13 rules in table- 2 .
Table-2 Fuzzy Rule Set

\begin{tabular}{|l|l|l|l|l|}
\hline Rule & $\begin{array}{l}\text { Work } \\
\text { Load }\end{array}$ & $\begin{array}{l}\text { CPU } \\
\text { Speed }\end{array}$ & Distance & $\begin{array}{l}\text { Node } \\
\text { State }\end{array}$ \\
\hline 1 & Low & Low & Low & LS \\
\hline 2 & Low & High & Low & SC \\
\hline 3 & Low & High & Medium & LS \\
\hline 4 & Low & Low & High & NS \\
\hline 5 & Low & High & High & LS \\
\hline 6 & Medium & High & Low & SC \\
\hline 7 & Medium & High & Medium & LS \\
\hline 8 & Medium & Low & Medium & NS \\
\hline 9 & Medium & Low & High & NS \\
\hline 10 & High & Medium & Low & NS \\
\hline 11 & High & Medium & Medium & NS \\
\hline 12 & High & High & Low & LS \\
\hline 13 & High & Low & High & NS \\
\hline
\end{tabular}

In table-2, SC denotes schedulable state, NS stands for not schedulable state and LS represents the state of likely to be scheduled. Among 13 rules, we intend to explain the following two cases,

(1) If $($ Work Load $=$ Low $\& \&$ CPU Speed $=$ High $\& \&$ Distance $=$ Low) Then

\section{"Node State is Schedulable" \\ End if}

(2) If (Work Load $=$ High $\& \&$ CPU Speed $=$ Low $\& \&$ Distance $=$ High) Then

"Node State is Not Schedulable"

End if

\subsubsection{Defuzzification}

A precise output value from fuzzy set is extracted during defuzzification phase. We have enormous methods for an efficient defuzzification. From those we choose to utilize the weighted mean method. The formulation for defuzzification process is, [16]

$$
O^{*}=\sum \eta_{o}(\bar{O}) / \sum \eta_{O}(\bar{O})
$$

where, $O^{*}$ is the derived output value, $\eta_{O}(\bar{O})$ denotes the strength of output membership function and $\bar{O}$ is the centroid of membership function.

\subsubsection{Task Allocation}

As a result of fuzzy logic, server nodes (S's) will be in the following three states as schedulable (SC), not schedulable (NS) and likely to be schedulable (LS). While allocating tasks, the $\mathrm{CH}$ checks task length of non-flexible tasks and compare the buffer length of $\mathrm{S}$ nodes in schedulable state. Then, $\mathrm{CH}$ allocates nonflexible tasks to $\mathrm{S}$ nodes in schedulable state. When number of $\mathrm{S}$ nodes in schedulable state is less than task length of non-flexible 
tasks, then the $\mathrm{CH}$ looks for nodes in likely to be scheduled (LS) state. Once, the allocation of non-flexible tasks are gets over, the $\mathrm{CH}$ goes for flexible tasks. The task allocation strategy is given in algorithm-1.

\section{Algorithm-1}

1. Let $\mathrm{S}$ be the set of server nodes and $\mathrm{C}$ be the set of client nodes

2. Consider nTi be the set of non-flexible tasks and fTi be the set of flexible tasks.

3. $\mathrm{C}$ node receives a new task

4. $\mathrm{C}$ transmits task details to $\mathrm{CH}$

5. $\mathrm{CH}$ looks for flexible field

6. If (flexible field $=1$ ) Then

6.1 The task is flexible

6.2 Else if (flexible field $=0$ ) Then

6.3 The task is non-flexible

7. End if

8. For (non- flexible tasks)

8.1 CH collects work load, CPU speed and distance informations of S' nodes

8.2 $\mathrm{CH}$ forwards these informations into fuzzy system

8.3 Nodes are separated into three states as SC, LS and NS

8.4 $\mathrm{CH}$ compares nTi task length with buffer availability of S' nodes

8.4.1 If (task length (nTi) < S (buffer availability)) Then

8.4.1.1. Allocates nTi among S' nodes in SC state

8.4.2 Else if (task length (nTi) > S (buffer availability))

Then

8.4.1.2 Allocate nTi among S' nodes in LS state

\subsubsection{End if}

9. For (flexible tasks)

9.1 CH allocates tasks among S' nodes in LS state

Our proposed technique is well suited for all real time applications, where buffer capacities and number tasks to be computed vary over time. This technique schedules the tasks adaptively and improves the quality of network. Using fuzzy logic system, this technique provides accurate load balancing and scheduling.

\section{SIMULATION RESULT}

\subsection{Simulation Model and Parameters}

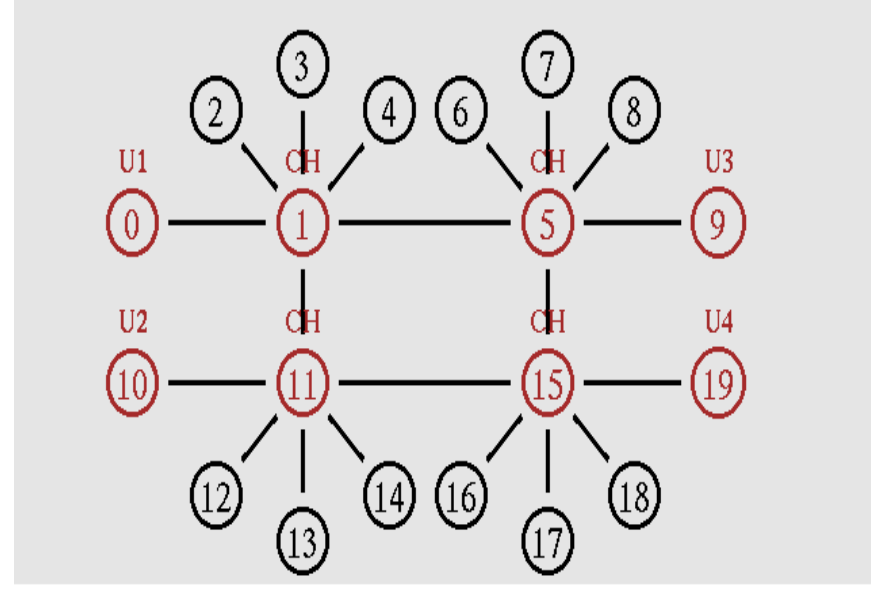

Figure: 6. Simulation Setup

In this section, we examine the performance of our Fuzzy based Load Balancing and Task Scheduling Technique (FLBTS) with an extensive simulation study based on NS-2 [17]. The simulation topology is given in Figure 6. We compare our results with QBS(Queue Based Scheduling) with normal scheduling [11].Various simulation parameters are given in table 3 .

Table: 3 Simulation Settings

\begin{tabular}{|l|l|}
\hline Mobile Nodes & 12 \\
\hline Users & 4 \\
\hline Area Size & $1000 \mathrm{X} 1000$ \\
\hline Mac & 802.11 \\
\hline Radio Range & $250 \mathrm{~m}$ \\
\hline Simulation Time & $50 \mathrm{sec}$ \\
\hline Traffic Source & $\mathrm{CBR}$ \\
\hline Packet Size & 512 \\
\hline Rate & $1,1.5,2,2.5$ and 3 Mb \\
\hline
\end{tabular}

\subsection{Performance Metrics}

In our experiments, we measure the following metrics.

Scheduling delay: It measures the average delay occurred while scheduling given tasks.

Throughput: It is the amount of work received in terms of $\mathrm{Mbit} / \mathrm{s}$ at the receiver.

Data Loss: It is the total number of data packets dropped at the receiver side.

Success Ratio: It is the ratio of number of tasks completed successfully to the total number of requested tasks.

\subsection{Results}

\section{A. Based on Load}

In the initial experiment, we vary the work load of the nodes by varying the size of the tasks as $1,1.5,2,2.5$ and $3 \mathrm{Mb}$. 


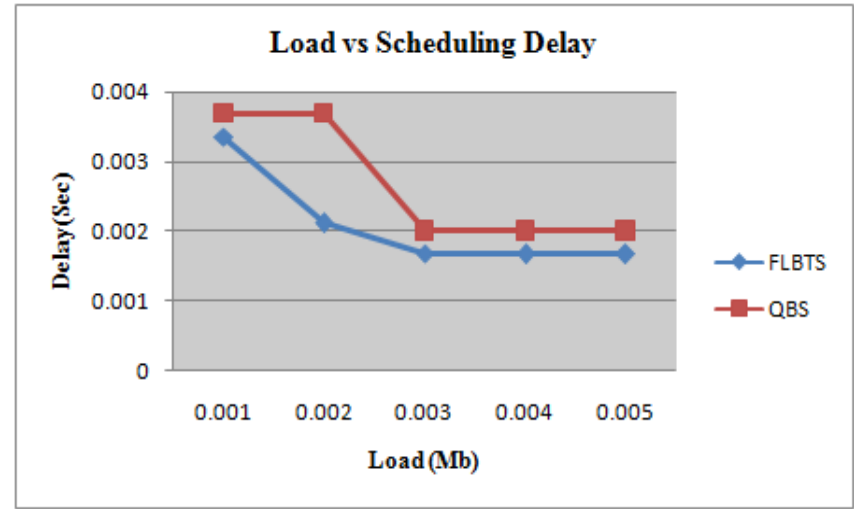

Figure 7: Load Vs Delay

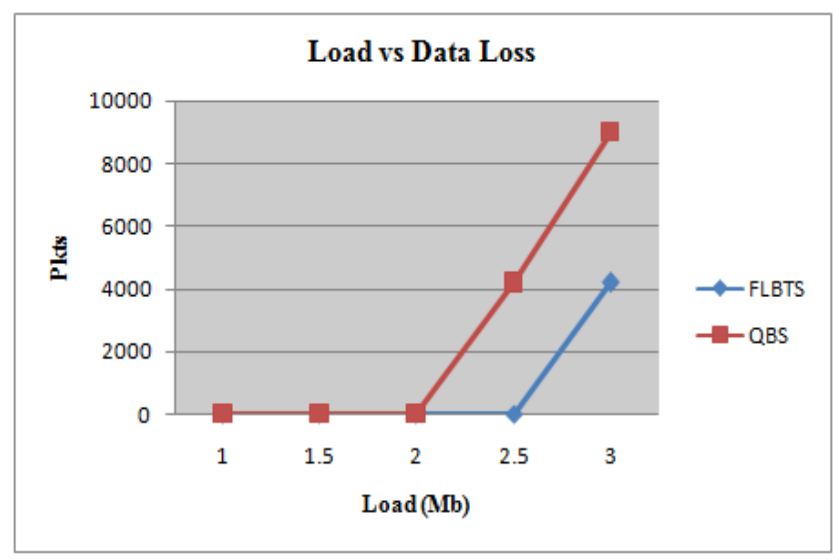

Figure 8: Load Vs Data Loss

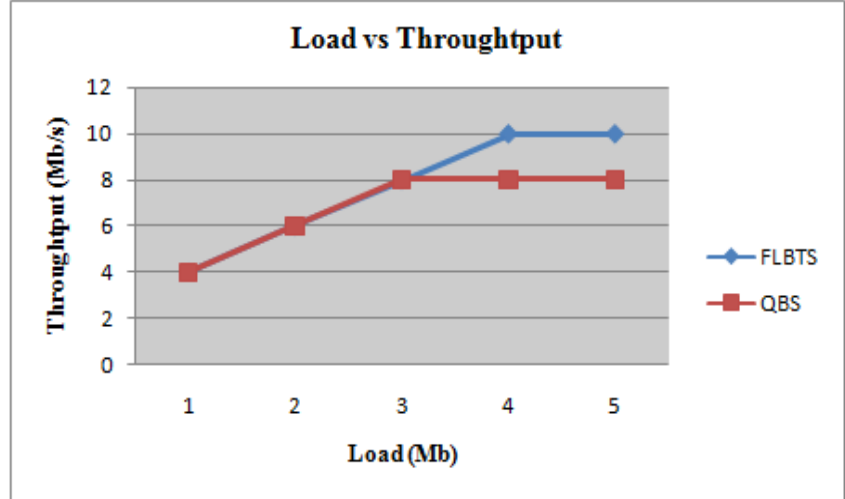

Figure 9: Load Vs Throughput

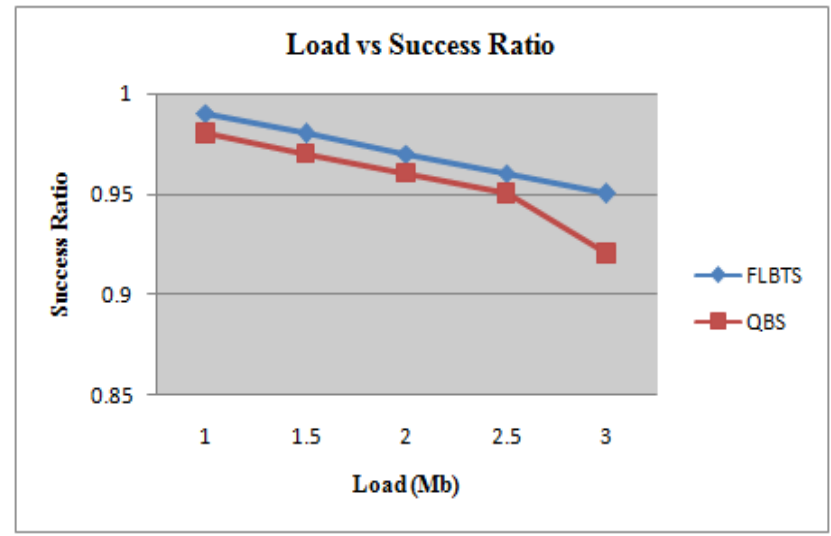

Figure10. Load Vs Success ratio
Figure 7 and 8 shows that delay and packet drop of FLBTS is significantly less than the existing QBS technique respectively.

Figure 9 and 10 shows that the throughput and success ratio of FLBTS is higher than the existing QBS method respectively.

\section{B. Based on Users}

In our second experiment, we vary the number of users from 1 from 4 each requesting variable number of tasks with size $1 \mathrm{Mb}$.

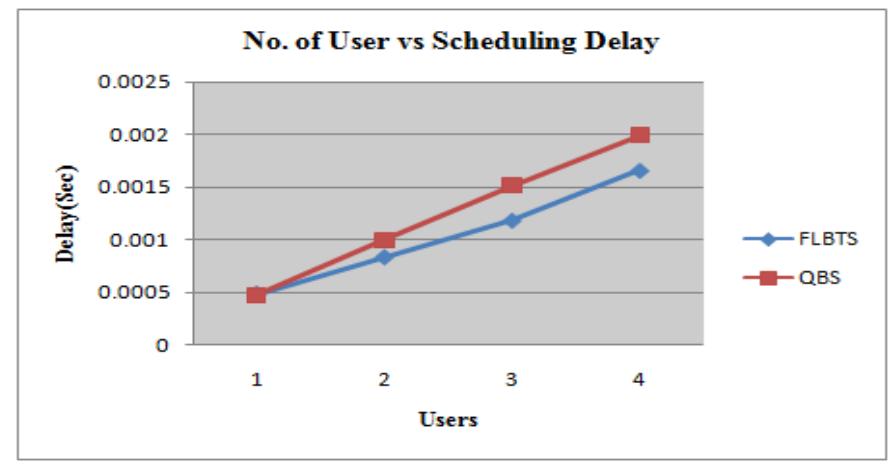

Figure 11: Users Vs Delay

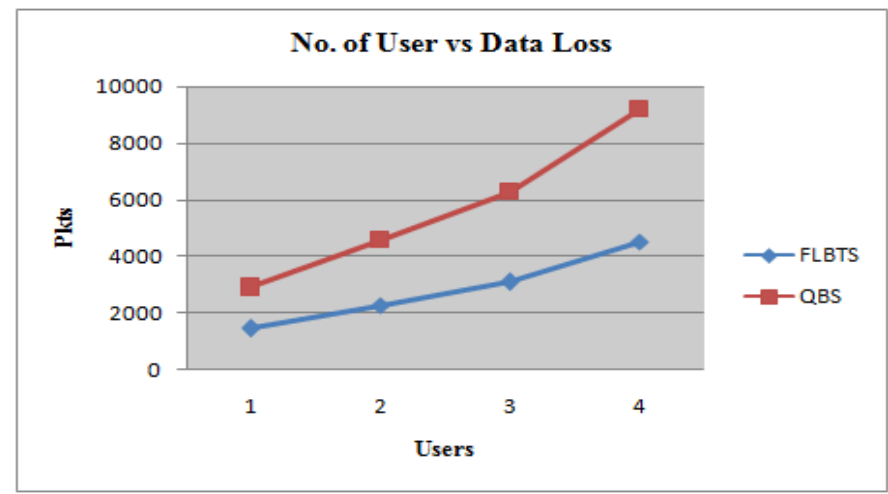

Figure 12: Users Vs Drop

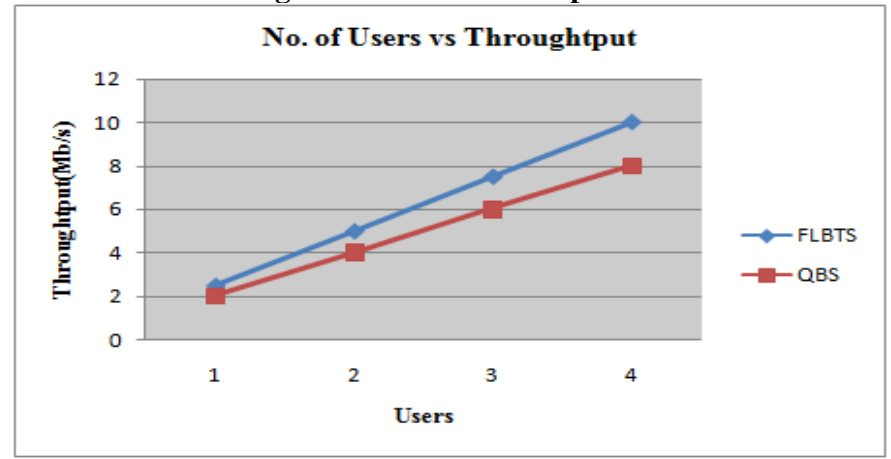

Fig 13: Users Vs Throughput

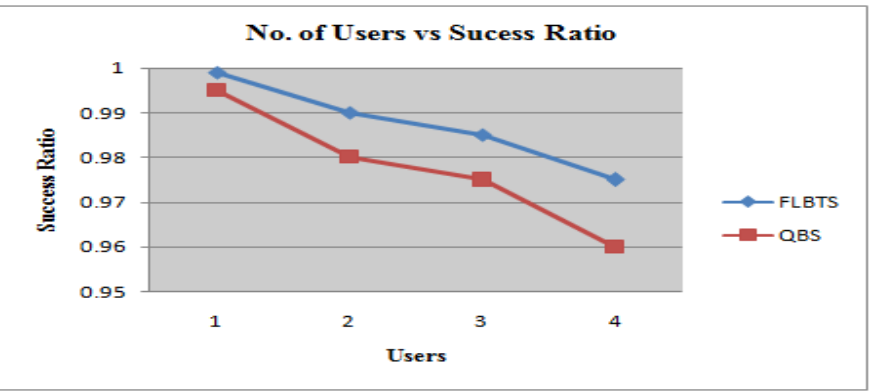

Fig 14: Users Vs Success Ratio 
Figure 11 and 12 shows that delay and packet drop of FLBTS is significantly less than the existing QBS technique respectively.

Figure 13 and 14 shows that the throughput and success ratio of FLBTS is higher than the existing QBS method respectively.

\section{CONCLUSION}

Load balancing \& task scheduling are old problem. But new solutions are required in modern distributed computing system. Load balancing \& task scheduling algorithm plays very important role in distributed system for managing load, and assigning them to appropriate resources. An efficient load balancing \& task scheduling algorithm can reduce the total execution time and increases throughput of system. In this paper, we have proposed a fuzzy based load balancing and task scheduling technique in distributed systems to improve the performance of distributed system. Fuzzy logic technique has very much potential in solving load balancing $\&$ task scheduling problem. Fuzzy logic make absolute output from uncertainties input. Our technique dynamically handles scheduling and load balancing at hand. The simulation result shows that efficiency of our technique is better than existing QBS technique. In future we will present new load balancing \& task scheduling technique using genetic algorithm \& neuro-fuzzy technique.

\section{REFERENCES}

[1] Daniel Grosu, Anthony T. Chronopoulos and Ming-Ying Leung, "Load Balancing in Distributed Systems: An Approach Using Cooperative Games", Proceedings of the 16th International Parallel and Distributed Processing Symposium, (IPDPS'02), 2002.

[2] Veeravalli Bharadwaj, Depasish Ghose and Thomas G. Robertazzi, "Divisible Load Theory: A New Paradigm for Load Scheduling in Distributed Systems", Journal of Cluster computing, Vol-6, Issue-1, 2003.

[3] Krishna Nadiminti, Marcos Dias de Assunção, and Rajkumar Buyya, "Distributed Systems and Recent Innovations: Challenges and Benefits", Grid Computing and Distributed Systems Laboratory, 2006.

[4] www.wikipedia.org.

[5] Sandeep Sharma, Sarabjit Singh, and Meenakshi Sharma, "Performance Analysis of Load Balancing Algorithms", World Academy of Science, Engineering and Technology, 2008

[6] Hisao Kameda, El-Zoghdy Said Fathyy and Inhwan Ryuz Jie Lix, "A Performance Comparison of Dynamic vs. Static Load Balancing Policies in a Mainframe \{ Personal Computer Network Model", Proceedings of the 39th IEEE Conference on Decision and Control, 2000.

[7] Daniel Grosua, Anthony T. and Chronopoulosb,"Noncooperative load balancing in distributed systems", Elsevier, Journal of Parallel and Distributed Computing, 2005.

[8] Chow KP and Kwok YK, "On load balancing for distributed multiagent computing", IEEE Transactions on Parallel and Distributed Systems, Vol- 13, pp- 787-801, 2002.
[9] Jiani Guo and Laxmi Narayan Bhuyan, "Load Balancing in a Cluster-Based Web Server for Multimedia Applications", IEEE Transactions On Parallel and Distributed Systems, Vol-17, 2006

[10] M. Nikravan and M. H. Kashani, "A Genetic Algorithm for Process Scheduling in Distributed Operating Systems Considering Load balancing”, Proceedings 21st European Conference on Modelling and Simulation (ECMS), 2007.

[11] Sivakumar Viswanathan, Bharadwaj Veeravalli and Thomas G. Robertazzi, "Resource-Aware Distributed Scheduling Strategies for Large-Scale Computational Cluster/Grid Systems", IEEE Transactions on Parallel and Distributed Systems, Vol-18,

[12] Michael Isard, Vijayan Prabhakaran, Jon Currey, Udi Wieder, Kunal Talwar and Andrew Goldberg, "Quincy: Fair Scheduling for Distributed Computing Clusters", Proceedings of the ACM SIGOPS 22nd symposium on Operating systems principles (SOSP '09), 2009.

[13] Xuan Lin, Ying Lu, Deogun, J. and Goddard, "Real-Time Divisible Load Scheduling for Cluster Computing", Proceedings of 13th IEEE Real Time and Embedded Technology and Applications Symposium, (RTAS '07), 2007.

[14] Kento Aida and Henri Casanova, "Scheduling MixedParallel Applications with AdvanceReservations", Proceedings of the 17th international symposium on High performance distributed computing (HPDC '08), 2008.

[15] Ananda Basu1, Saddek Bensalem, Doron Peled, and Joseph Sifakis," Priority Scheduling of Distributed Systems Based on Model Checking", Proceedings of the 21st International Conference on Computer Aided Verification (CAV '09), 2009.

[16] Zuo Jing, Chi Xuefen, Lin Guan and Li Hongxia, "Serviceaware Multi-constrained Routing Protocol with QoS Guarantee Based on Fuzzy Logic", IEEE 22nd International Conference on Advanced Information Networking and Applications - Workshops, (AINAW), pp- 762 - 767, 2008.

[17]NetworkSimulator:http:///www.isi.edu/nsnam/ns

\section{AUTHOR'S PROFILE}

Abhijit Rajguru received the B.E and M.Tech degree in Computer Science \& Engineering, from Shivaji University, Kolhapur, Maharashtra(INDIA) in 2007 and 2009, respectively. $\mathrm{He}$ is research scholar at WIT, Solapur (Solapur University, Maharashtra, INDIA). His research interest includes Distributed System, cloud computing, grid computing, load balancing.

Dr. Mrs. S. S. Apte received Ph.D. degree in Computer Engineering. She having 33 years teaching experience and 02 years industry experience. She is working as professor in CSE department in WIT Solapur. Her research interest includes Computer architecture, distributed system, image processing. 